\title{
GLAD!
}

Revue sur le langage, le genre, les sexualités

$05 \mid 2018$

Raconter les sexualités depuis la marge

\section{Premiers jets}

Splashes

\section{ArianE Sirota}

\section{(2) OpenEdition}

Journals

Édition électronique

URL : http://journals.openedition.org/glad/1370

ISSN : 2551-0819

Éditeur

Association GSL

Référence électronique

ArianE Sirota, «Premiers jets », GLAD! [En ligne], 05 | 2018, mis en ligne le 15 décembre 2018, consulté le 17 décembre 2020. URL : http://journals.openedition.org/glad/1370

Ce document a été généré automatiquement le 17 décembre 2020.

\section{(c) (i) (9)}

La revue GLAD! est mise à disposition selon les termes de la Licence Creative Commons Attribution Pas d'Utilisation Commerciale - Pas de Modification 4.0 International. 


\title{
Premiers jets
}

\author{
Splashes
}

\section{ArianE Sirota}

1 Jouer au bricolo m'amuse toujours plus chez les autres. Mon propre chantier n'en finit jamais et je ne me remercie pas, ne sais pas me réjouir des quelques pas franchis chaque fois - quand je veux bien m'y remettre. Mes outils restent dans leur boîte ou pas loin, prêts à être utilisés ailleurs.

2 Depuis le temps que ma famille use le parquet flottant des rayons d'Ikea, il y a longtemps que je ne lis plus méthodiquement les notices de montage. Son meuble a beau venir d'une autre enseigne, la quincaillerie et les perçages caractéristiques sont les mêmes, un simple jeu de construction pour moi. Puisque c'est l'un de mes rares sujets de fierté, être à la hauteur de mes annonces était crucial, et faire vite faisait partie de mes objectifs, je n'ai prêté aucune attention à ma sécurité : j'ai laissé traîner des outils, déplacé de grosses pièces à bout de bras et j'en passe. Mon corps n'était pas d'accord,

3 Iel est entréE, dans la salle de bain, dans la baignoire. Sans un mot, sans un regard direct. Son mécontentement seulement reflété par le miroir. Moi qui voulais elui faire plaisir... Je soupire au toucher de son bassin contre mes fesses. Iel me prend la fleur de douche déjà mousseuse et la passe lentement sur ma peau, me pousse. Je m'appuie contre la faïence, la robinetterie me renvoie des reflets qui rappellent les contorsions consenties au maintien des premières pièces pour les assembler. Iel me tient à distance, passe son bras entre mes jambes, se saisit de la pomme et me rince, toujours avec cette lenteur inhabituelle.

4 Ses gestes saccadés, familiers, refont surface une fois l'eau coupée. Iel ne met pas dix secondes à sortir de la baignoire, se sécher avant de me jeter une serviette à la figure et disparaître. Je me dirige vers sa chambre dès que je ne goutte plus. Le lit est fait, ma boîte est fermée, près de la porte. Je ne vois pas mon sac, celui qui contient mes vêtements propres. Iel me pousse de l'épaule.

5 «Alors, tu croyais qu't'allais pouvoir m'baiser après ça ? Genre t'as payé d'avance pour ton plaisir? T'es toutE nouéE maintenant, pleinE de courbatures qui te gêneront peut- 
être pendant plusieurs jours... Et tu crois que ça m'fait plaisir ? Hein, tu crois quoi ? Que j'avais besoin de toi pour le faire ? Comme si j'avais eu besoin d'un homme ?! »

6 Iel me pousse sur le lit, le drap de bain s'ouvre dans le mouvement que j'esquisse pour accompagner ma chute. J'attends la suite, mes appréhensions éclipsées par l'excitation.

7 «Ne me regarde pas!»

8 À peine suis-je sur le ventre qu'une main claque mon postérieur. Iel s'approche, me pétrit. Une nouvelle fessée, à droite cette fois, me tire un gémissement - iel sait ce côté particulièrement sensible et en profite. J'ai été vilainE de penser à sa place, faire à sa place, même en pensant elui faire plaisir. Entre nous, c'est une circonstance aggravante. J'ai été si vilainE que je ne mérite pas ses soins déguisés en punition. L'envie de læ supplier monte pourtant, tonitrue dans ma tête, tourne dans ma bouche derrière des lèvres que je peine à garder serrées. Laisser la main, découvrir ce qu'iel me réserve. Froid, le lubrifiant sur mon anus, alors que la chaleur n'a pas cessé d'augmenter au niveau des rondeurs voisines.

9 Je ne reconnais pas l'objet glissé entre elles; un nouveau jouet dans cette maison? Sa longueur ne fait pas refluer l'envie de me coller contre l'amantE. Mon bassin en prend le chemin, sa main se glisse dessous et commence à tapoter mon mont; des séries irrégulières, appliquées juste au sommet à fréquence et intensité croissantes. Il ne m'avait pas été facile de dire ma difficulté à gérer ce stimulus qui tire sur la corde de mon désir, alors que sa présence suffit à la tendre vers un registre aigu. J'elui étais reconnaissantE de me l'avoir épargné, jusqu'à aujourd'hui, quand iel ajoute à ses suites percussives deux nouvelles notes: la toison empoignée et un doigt passé entre les nymphes.

10 J'ai perdu le compte des renflements qui ont passé et repassé mon sphincter. Je tangue, puis m'écroule, profil et épaule droits enfoncés dans le matelas. J'ai chaud, nous atteignons un palier inconfortable, je læ supplie de m'emplir et me retourne pour elui faciliter la tâche, vulve luisante, exposée. Je découvre son harnais orné d'un gode de taille respectable. Iel relève mes cuisses et s'approche enfin. Ses mouvements sont amples, le silicone bute tout au fond à chaque balancement. Il faut que j'abdique pour y trouver du plaisir, iel le sait et connait le rythme qui m'aide à m'abandonner. Mon col entre en résonance.

11 Son regard, un peu dur, plonge enfin dans le mien : iel se gargarise de son pouvoir, me force maintenant à ne pas détourner les yeux, d'une main fermée sur ma nuque alors que le flot d'émotions me déborde. J'imagine mes larmes former une constellation de tâches parfaitement rondes sur les draps, sous ma tête, sous ses doigts. C'est peut-être le pire aspect du supplice du jour. La libération de toutes les tensions dans mon corps et entre nous, je la souhaitais, mais passer par tous ces états sous ses yeux... Iel fond sur ma bouche un instant avant de la laisser s'ouvrir sur ma voix secrète.

Enfin l'unE contre l'autre. Son souffle court me fait frissonner sous la sueur. Je n'ose pas læ serrer dans mes bras, ce privilège n'est peut-être pas encore offert. Doucement, iel se retire en souriant des fluides qui nous lient, en recueille de sa langue. Mon bassin pivote vivement, cherchant à se dérober par un mouvement réflexe écourté : tous mes orifices n'ont pas été libérés. Pendant qu'iel se déharnache, je trouve une position dans laquelle me détendre. À peine les cliquetis ont-ils cessé que je sens sa respiration dans mes replis trempés. Sa bouche se fraie un chemin alors qu'iel sort les perles une à une. Quand plus rien ne chatouille mon périnée, sa langue remonte vers mon clitoris, nous 
sommes loin d'une douceur postliminaire. Mon bassin tressaille vivement par moments, iel s'y est vigoureusement arriméE. L'étreinte se desserre autour de ma cuisse gauche, sa main droite, gantée, glisse en moi sans effort. Son poing à peine fermé oscille, presse rythmiquement la paroi antérieure. L'amantE halète plus qu'iel ne me suce à présent et se redresse, délaissant ma hanche pour se caresser. Iel me tient pourtant, me tire vers elui de son poing autour duquel je m'applique à me refermer. Mon autre voix emplit la chambre, je suis toutE entierE dans ce son. Jusqu'à ce qu'un autre bruit résonne. Un bruit humide. Trois coups, courts, l'ont touchéE en plein cœur, son tatouage brille sous l'éjaculat répandu sur son buste.

C'est moi qui ai fait ça?

Nous.

\section{RÉSUMÉS}

Premiers jets a connu plusieurs versions. La première a été publiée dans Cyprine, sexe désir et capitalisme, fanzine de la Radicale Queer Semaine de Montréal 2012. La deuxième en 2015, au sein du recueil Projet $Q, 15$ nouvelles érotiques lesbo-queer chez Des ailes sur un tracteur, maison d'édition disparue depuis. La voici revue aujourd'hui avec la complicité des éditions Pousse-pied.

Splashes has been rewritten several times. Its first publication was in Cyprine, sexe désir et capitalisme, the Radicale Queer Semaine of Montreal's fanzine in 2012. The second was in the miscellany Projet Q, 15 nouvelles érotiques lesbo-queer published by Des Ailes sur un tracteur, which closed since. Here in GLAD! a revision due to the current partnership between the author and the publishing house Pousse-pied.

\section{INDEX}

Thèmes : Créations

Mots-clés : queer, langue, sexe, littérature

Keywords : queer, language, sex, literature

\section{AUTEUR}

\section{ARIANE SIROTA}

PlasticienNE et auteurice genderqueer, ArianE Sirota questionne les représentations pour entrouvrir des brèches propices au débridage des imaginaires. Son premier roman, Foyer, paru en 2017, met en scène un réseau d'utopies féministes, chacune ayant un habitat, une organisation sociale et un langage différents. 